\title{
Antineutrino Flux from the Laguna Verde Nuclear Power Plant
}

\author{
Marisol Chavez-Estrada and Alexis A. Aguilar-Arevalo \\ Instituto de Ciencias Nucleares, Universidad Nacional Autónoma de México, 04510 México, DF, Mexico \\ Correspondence should be addressed to Marisol Chavez-Estrada; marisol.chavez@correo.nucleares.unam.mx
}

Received 29 August 2015; Revised 4 November 2015; Accepted 16 November 2015

Academic Editor: Luis A. Anchordoqui

Copyright (C) 2015 M. Chavez-Estrada and A. A. Aguilar-Arevalo. This is an open access article distributed under the Creative Commons Attribution License, which permits unrestricted use, distribution, and reproduction in any medium, provided the original work is properly cited. The publication of this article was funded by SCOAP ${ }^{3}$.

\begin{abstract}
We present a calculation of the antineutrino flux produced by the reactors at the Laguna Verde Nuclear Power Plant in México, based on the antineutrino spectra produced in the decay chains of the fission fragments of the main isotopes in the reactor core, and their fission rates, which have been calculated using the DRAGON simulation code. We also present an estimate of the number of expected events in a detector made of plastic scintillator with a mass of $1 \mathrm{ton}$, at $100 \mathrm{~m}$ from the reactor cores.
\end{abstract}

\section{Introduction}

In recent years, there has been significant progress in understanding the properties of neutrinos; most notably, since 2012, the results of the reactor neutrino experiments, Daya Bay [1], Reno [2], and Double Chooz [3], have determined that the mixing angle $\theta_{13}$ is nonzero with a high level of significance. The current degree of development of neutrino detection technology is close to making a reality to nonintrusively monitor the operational status, power level, and fissile content of a nuclear reactor in real time using detectors placed at distances of a few tens of meters.

For a technological application such as reactor monitoring, only antineutrinos with energies above the threshold of the Inverse Beta Decay (IBD) reaction (see (1)) on free protons $\left(E_{v}>1.8 \mathrm{MeV}\right)$ can be realistically considered at present:

$$
\bar{\nu}_{e}+p \longrightarrow e^{+}+n \text {. }
$$

IBD interactions of neutrinos on free protons can be identified through the correlation of the prompt positron signal and a delayed neutron capture signal in liquid or plastic scintillator-based detectors. In general, the spectrum of the antineutrinos from a nuclear reactor detected via IBD has a peak between 3.5 and $4 \mathrm{MeV}$. Since the number of emitted antineutrinos and their average energy depend on the amounts of ${ }^{235} \mathrm{U},{ }^{238} \mathrm{U},{ }^{239} \mathrm{Pu}$, and ${ }^{241} \mathrm{Pu}$ isotopes present in the core, the measured energy spectrum provides a direct image of the fuel composition of the reactor core at a given time.

Monitoring nuclear reactors through their antineutrino flux is a complimentary and promising new tool for supervising the operations of nuclear plants, which are bound to operate according to protocols established by the International Atomic Energy Agency (IAEA). This agency is responsible for ensuring that nuclear reactors worldwide operate legally, preventing the diversion of fissile material to activities which could lead to the manufacture of weapons.

Many research groups worldwide [4-10] have studied this application aiming at adding antineutrino detection to the techniques used to implement reactor safeguards. In a medium term plan (5 to 8 years), IAEA [11] has proposed that antineutrino detectors for safeguard applications should be able to provide information on the thermal power, fissile content, and operational status of reactors while deployed aboveground in a compact volume like that of a standard $12 \mathrm{~m}$ ISO container, to minimize its intrusiveness with the nuclear power plant operations.

One of the IAEA's safeguards goals is to demonstrate the ability to detect the unauthorized removal of as little as $8 \mathrm{~kg}$ of plutonium (defined as a "significant quantity") from a nuclear reactor within a 3-month period [12]. According to the agency, it is not possible to exclude the possibility that an explosive nuclear device is manufactured having this amount of material unaccounted for over this time. 
The accuracy to which the plutonium content in a nuclear reactor can be measured using neutrinos has been the subject of a number of studies [13-16]. One method [16], albeit with reduced sensitivity compared to IAEA's "significant quantity," consists of measuring the time evolution of the antineutrino count rate in a detector. Adding evolution of antineutrino spectrum shape as well as combination with standard IAEA safeguards methodologies may eventually allow reaching the desired goal set by the agency. Recent studies suggest that the observation of shape distortions with sufficient significance could be achieved with realistic energy resolutions [8] ( 12\% at $2 \mathrm{MeV}$ neutrino energy).

A recent demonstration that the ON/OFF cycle, thermal power stability, and fissile inventory of a reactor can be measured from the total antineutrino event rate in a compact $\left(\sim 1 \mathrm{~m}^{3}\right.$ scale) and simple detector was given by the SONGS1 experiment [4], which used a gadolinium- (Gd-) loaded liquid scintillator detector, deployed under $25 \mathrm{MWe}$ overburden, at $25 \mathrm{~m}$ from the reactor core of the $3.46 \mathrm{GWth}$ San Onofre Nuclear Generating Station in Southern California in the United States. The experiment estimated a sensitivity to the removal of $\sim 70 \mathrm{~kg}$ of ${ }^{239} \mathrm{Pu}$ with $95 \% \mathrm{CL}$.

Another recent development is the PANDA $[5,6]$ experiment, which used a segmented plastic scintillator detector located at ground level, with modules wrapped in Gd-coated sheets, at $36 \mathrm{~m}$ from Unit 2 reactor at the Ohi Power Station in Japan. This detector design uses event topology information to tag antineutrino events and to discriminate them from backgrounds such as fast neutrons, allowing operating it on the surface. Furthermore, it can be transported and operated easily inside a compact vehicle and has the advantage that, unlike the liquid scintillators, it is nonflammable. Studies from a $360 \mathrm{~kg}$ prototype have shown promise that this detector technology could be further developed to achieve the IAEA medium term goals.

México has two nuclear fission reactors at the Laguna Verde Nuclear Power Plant (LVNPP), which has been operating commercially since 1990 (Unit 1) and 1995 (Unit 2). This is the only nuclear power plant in the country and it generates about $5 \%$ of its total electric power production. In this work, we calculate the antineutrino flux produced by these reactors and give some estimates of the number of events expected in a generic plastic scintillator detector located at a distance of $100 \mathrm{~m}$ from the reactor cores. Knowledge of the antineutrino flux produced by a particular reactor is an important step towards its monitoring using this method.

While abundant literature exists about the calculation of neutrino fluxes from pressurized water reactors (PWR) [21-28], much less information is available about neutrino production at boiling water reactors (BWR) (see, e.g., [29]). These two reactor types operate under the same basic physical principles regarding nuclear fission, but differences in their operational procedures may affect differently the neutrino flux they produce, as well as its evolution along the reactor fuel cycle.

This paper is organized as follows. In Section 2, main technical data on the LVNPP and its reactors are detailed. In Section 3, a description about the production of antineutrinos from nuclear reactors is given, and the calculation of antineutrino flux is presented. In Section 4, parameters and characteristics of the DRAGON simulation code used to calculate fission rates of four major fissile isotopes for this paper are described. Section 5 shows the event rates or interactions that are expected to be observed with a 1-ton plastic scintillator detector placed at 100 meters from the LVNPP reactors. Finally, Section 6 presents the conclusions.

\section{The Laguna Verde Nuclear Power Plant (LVNPP)}

The LVNPP is located in the municipality of Alto Lucero, Veracruz, México. This plant has two twin units, each equipped with a BWR-5 second-generation reactor with a Mark II containment design, supplied by General Electric with a capacity of $2027 \mathrm{MWth}$, and net electrical output of 805 MWe. Both reactors operate with enriched uranium as fuel and demineralized water as moderator and coolant. It is the only nuclear power plant in the country and generates about $5 \%$ of the total electric power production in México [30].

Each nuclear reactor core can be approximately represented by a cylinder $\sim 4 \mathrm{~m}$ in height and $4 \mathrm{~m}$ in diameter containing 444 fuel assemblies and 109 control bars arranged as shown in Figure 1. A fuel assembly is a square prism with sides of $\sim 13 \mathrm{~cm}$ and $4 \mathrm{~m}$ in height, containing an array of fuel rods (configurations of $8 \times 8,10 \times 10$, or $12 \times 12$ have been either used or considered). Within a given fuel assembly, some of the rods contain uranium oxide $\left(\mathrm{UO}_{2}\right)$ with various levels of enrichment, others contain a mixture of $\mathrm{UO}_{2}$ with gadolinium oxide $\left(\mathrm{Gd}_{2} \mathrm{O}_{3}\right)$ at varying concentrations, and some of them are hollow in order to allow the flow of the refrigerant through them. Combining rods with different levels of enrichment and $\mathrm{Gd}_{2} \mathrm{O}_{3}$ concentrations permits adjusting the properties of the medium for the production, absorption, and diffusion of neutrons differently at various location across the core.

\section{DRAGON Simulation of the Reactor Core}

We used the numerical simulation code DRAGON [31] with the modifications described in [32] to implement a simulation for the LVNPP reactors and extract the time evolution of the fission rates (number of fission instances per second) of each of the main fissile isotopes in the core. Several research groups worldwide have implemented this simulation for PWR reactors, aiming at the same objective [33]. The code solves the neutron transport equations of individual fuel assemblies, whose detailed composition is given as an input. The overall behaviour of neutrons across the core can be calculated by summing over the various types of assemblies that compose a given core configuration.

As described in Section 2, a geometry with 444 fuel assemblies with 3 different types of ${ }^{235} \mathrm{U}$ enrichment was considered. The initial fuel load that was assumed in this work is that shown in Figure 1. Note that in this model no assembly 

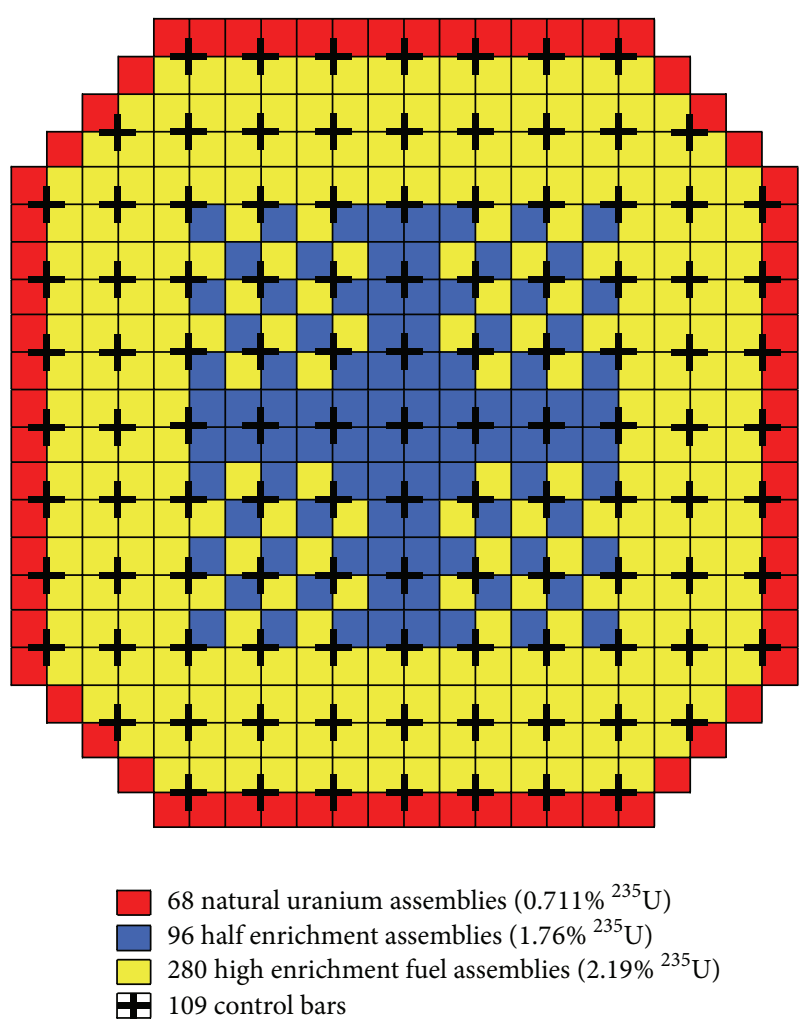

FIGURE 1: Schematic of a cross section of one of the LVNPP reactor cores [17]. The "red" assemblies $(0.711 \%)$ are located on the perimeter of the reactor core, while the "blue" assemblies (1.76\%) are arranged in the central area. There are 109 control bars represented as crosses in the figure.

contains plutonium at the beginning of the operation cycle.

In Figure 2, we illustrate a single fuel assembly (in this case with a $10 \times 10$ rod configuration) [34] corresponding to one with average enrichment of $1.76 \%$ (blue assembly). The dimensions of a single unit cell (the place occupied by a single fuel rod) are shown in Figure 3. The three types of assembly (red, yellow, and blue) share the same geometry; however, the composition of the fuel rods within a given assembly is, in general, different. The number of assemblies of each type, $N_{A}$, is $N_{\text {red }}=68, N_{\text {yellow }}=280$, and $N_{\text {blue }}=96$, totaling 444 .

We considered eight different fuel rod compositions, labeled $C X$, with $X=0,1,2,3,4,5,6, \mathrm{G}$, and used them to construct the various assemblies with the desired ${ }^{235} \mathrm{U}$ enrichment and gadolinium concentration. Only fuel rods of type $\mathrm{G}$ contain gadolinium. Table 1 shows the number of fuel rods of each type which are used to build each of the three assemblies. As an example, Table 2 shows how we constructed the blue assembly.

Table 3 lists the values used for the various parameters required by the simulation. Tests showed small dependence of the fission rates on variations of the coolant, moderator, and fuel temperatures, while maintaining the thermal power constant.

A simulation was run for each of the three types of assembly $A$ (red, blue, or yellow in Figure 1), providing
TABLE 1: Number of fuel bars of type $C X(X=0, \ldots, 6, G)$ for each assembly. There are a total of 92 bars for each assembly.

\begin{tabular}{lcccccccc}
\hline Assembly & C0 & C1 & C2 & C3 & C4 & C5 & C6 & CG \\
\hline Red & 92 & 0 & 0 & 0 & 0 & 0 & 0 & 0 \\
Blue & 59 & 0 & 0 & 3 & 4 & 6 & 8 & 12 \\
Yellow & 34 & 9 & 19 & 0 & 0 & 0 & 0 & 30 \\
\hline
\end{tabular}

TABLE 2: Isotopic composition of fuel bars for blue assembly in Figure $1 . \mathrm{UO}_{2}$ and $\mathrm{UO}_{2}-\mathrm{Gd}_{2} \mathrm{O}_{3}$ bars with overall average enrichment of $1.76 \%$ of ${ }^{235} \mathrm{U}$ (relative to total $\mathrm{U}$ ). Fuel rods with Gd admixture have enrichment of $5.2 \%$ (in total) and 3.792\% (relative to U).

\begin{tabular}{lcccccc}
\hline \multirow{2}{*}{ Rod type Number of rods } & $\begin{array}{c}{ }^{234} \mathrm{U} \\
(\%)\end{array}$ & $\begin{array}{c}{ }^{235} \mathrm{U} \\
(\%)\end{array}$ & $\begin{array}{c}{ }^{238} \mathrm{U} \\
(\%)\end{array}$ & $\begin{array}{c}\mathrm{O}_{16} \\
(\%)\end{array}$ & $\begin{array}{c}\mathrm{Gd} \\
(\%)\end{array}$ \\
\hline C0 & 59 & 0.005 & 0.627 & 87.517 & 11.852 & - \\
C3 & 3 & 0.029 & 2.468 & 85.651 & 11.852 & - \\
C4 & 4 & 0.029 & 2.821 & 85.298 & 11.852 & - \\
C5 & 6 & 0.029 & 3.173 & 84.945 & 11.852 & - \\
C6 & 8 & 0.029 & 3.482 & 84.637 & 11.852 & - \\
CG & 12 & 0.028 & 3.142 & 79.691 & 11.933 & 5.206 \\
\hline
\end{tabular}

TABLE 3: Parameters of the BWR core used in the simulation with DRAGON code [20].

\begin{tabular}{lc}
\hline Thermal power (of each reactor) & $2027 \mathrm{MWth}$ \\
\hline Specific power (of each reactor) & $20.43 \mathrm{MW} / \mathrm{ton}$ \\
\hline Moderator & Demineralized water \\
Temperature & $600 \mathrm{~K}$ \\
Density & $0.720 \mathrm{~g} / \mathrm{cm}^{3}$ \\
\hline Coolant & Demineralized water \\
Temperature & $400 \mathrm{~K}$ \\
Density & $0.720 \mathrm{~g} / \mathrm{cm}^{3}$ \\
\hline Fuel assemblies & 444 \\
Red assemblies & 68 \\
Blue assemblies & 96 \\
Yellow assemblies & 280 \\
\hline Single unit cell $(L \times L)$ & $1.295 \mathrm{~cm} \times 1.295 \mathrm{~cm}$ \\
\hline Fuel bars per assembly & 92 \\
Composition & $\mathrm{UO}_{2} / \mathrm{UO} \mathrm{O}_{2}-\mathrm{Gd}_{2} \mathrm{O}_{3}$ \\
Cladding & $\mathrm{Zircaloy} 2$ \\
Zircaloy density & $5.821 \mathrm{~g} / \mathrm{cm}^{3}$ \\
Zircaloy temperature & $600 \mathrm{~K}$ \\
Fuel temperature & $900 \mathrm{~K}$ \\
Fuel density & $10.079 \mathrm{~g} / \mathrm{cm}^{3}$ \\
Fuel bar radius $(R 1)$ & $0.438 \mathrm{~cm}$ \\
Zircaloy cladding radius $(R 2)$ & $0.513 \mathrm{~cm}$ \\
Fuel bar height & $400 \mathrm{~cm}$ \\
\hline Coolant pipes per assembly & 2 \\
Composition & $1.0 \mathrm{~cm}$ \\
Pipe radius (occupied by water) & $1.2 \mathrm{~cm}$ \\
Pipe radius (occupied by Zircaloy) & \\
\hline & \\
\hline
\end{tabular}




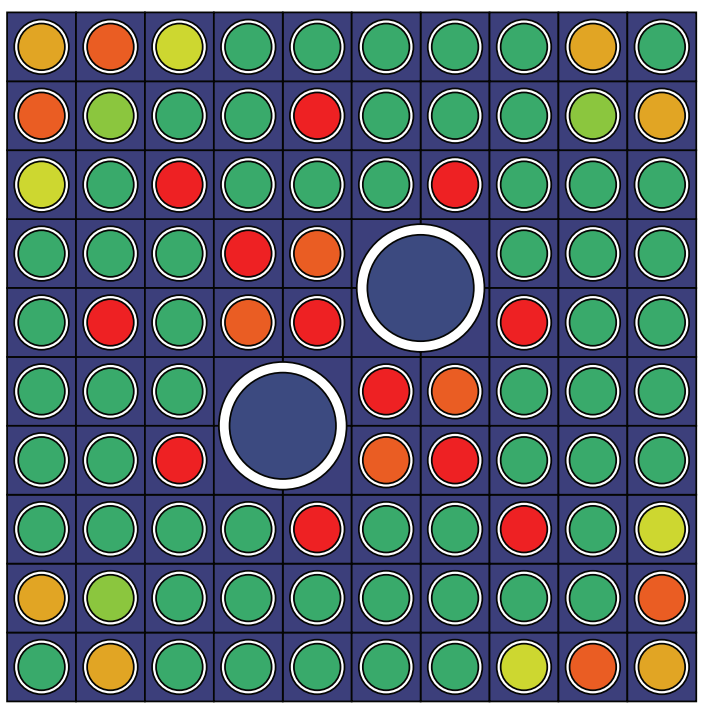

(a)

\begin{tabular}{|c|c|c|c|c|c|c|c|c|c|}
\hline C5 & C6 & C4 & $C 0$ & $C 0$ & $C 0$ & $C 0$ & C0 & C5 & C0 \\
\hline C6 & C3 & C0 & $C 0$ & CG & $C 0$ & C0 & C0 & C3 & C5 \\
\hline$C 4$ & C0 & CG & C0 & C0 & C0 & CG & C0 & C0 & C0 \\
\hline $\mathrm{CO}$ & C0 & C0 & CG & C6 & & & C0 & C0 & C0 \\
\hline C0 & CG & C0 & C6 & CG & & & CG & C0 & C0 \\
\hline C0 & $C 0$ & $\mathrm{CO}$ & & & $C G$ & C6 & C0 & C0 & $\mathrm{CO}$ \\
\hline $\mathrm{CO}$ & C0 & CG & & & C6 & CG & C0 & $\mathrm{CO}$ & $\mathrm{CO}$ \\
\hline C0 & $C 0$ & C0 & C0 & $C G$ & CO & C0 & CG & C0 & $C 4$ \\
\hline C5 & C3 & C0 & C0 & C0 & $\mathrm{CO}$ & C0 & C0 & $\mathrm{CO}$ & C6 \\
\hline $\mathrm{CO}$ & C5 & $\mathrm{CO}$ & $\mathrm{CO}$ & $C 0$ & $C 0$ & $\mathrm{CO}$ & C4 & C6 & C5 \\
\hline
\end{tabular}

(b)

FIGURE 2: Arrangement of fuel rods for blue assembly (half enrichment). (a) DRAGON code output. (b) Bar type (see Table 2). Notice that initially there are no fuel bars with $\mathrm{Pu}$.

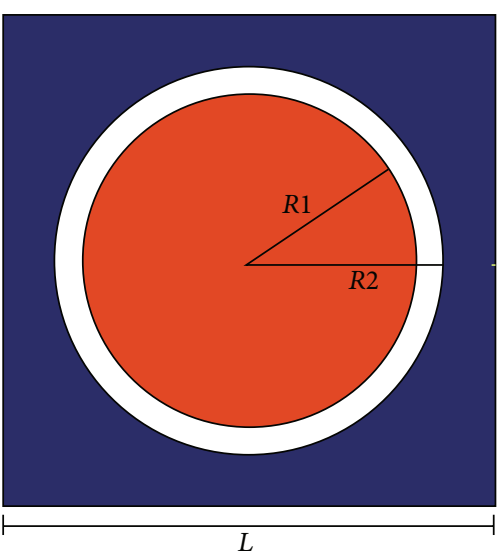

FIgURE 3: Cross section of a single unit cell. A fuel bar (in red) with Zircaloy cladding (white) immersed in demineralized water as moderator. Complete assembly is formed by 92 of these cells. The values for $R 1, R 2$, and $L$ are described in Table 3.

the fission rates $f_{i}^{(C X ; A)}$ for each type of fuel rod, and for each one of the fissile isotopes, $i=1,2,3,4\left({ }^{235} \mathrm{U},{ }^{238} \mathrm{U},{ }^{239} \mathrm{Pu}\right.$, and ${ }^{241} \mathrm{Pu}$, resp.).

To obtain the fission rates for a given assembly, $f_{i}^{A}$, we multiply the fission rate for each type of fuel rod times the number of rods of its type in the assembly, $N_{\text {rods }}$, and sum over all fuel rod types in the assembly; that is,

$$
f_{i}^{A}=\sum_{C X \in A} N_{\text {rods }}(C X ; A) f_{i}^{(C X ; A)} .
$$

Finally, to calculate the fission rates of isotope $i$ in the complete reactor core, $f_{i}$ (Figure 4 ), we multiply the number

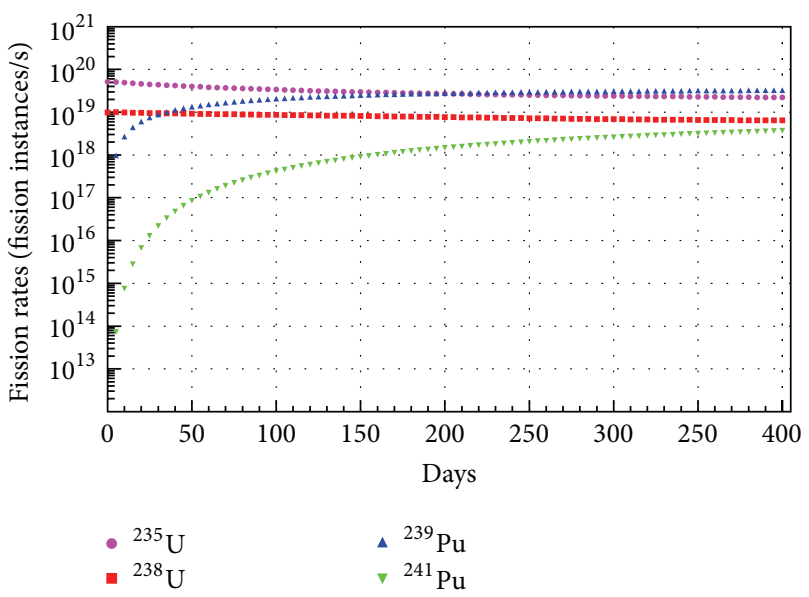

FIGURE 4: Fission rates (normalized) obtained with DRAGON for the 4 main fissile isotopes in the 444 fuel assemblies that make up the core of BWR-5 of 2.027 GWth like the LVNPP reactor.

of assemblies of each type times the corresponding fission rates for that assembly:

$$
f_{i}=\sum_{A} N_{A} f_{i}^{A}
$$

The simulation was run sequentially in time intervals of 5 days of evolution of the nuclear reactor core until completion of a full operation cycle of 400 days.

As is expected for an initial load without plutonium, the fission rates of ${ }^{239} \mathrm{Pu}$ and ${ }^{241} \mathrm{Pu}$ start at zero and rapidly grow to become comparable to those of the $\mathrm{U}$ isotopes within a few weeks of operation. A more realistic situation should consider that, between two fuel reloads, there will always be some remnant of plutonium inside the reactor, because when 
this action is performed, only a fraction of the spent fuel is replaced by new material.

\section{The Antineutrino Flux}

The power output of a nuclear reactor originates from the energy released in the fission of heavy elements, such as uranium and plutonium, into lighter fission fragments which are often unstable. The beta decay, $(A, Z) \rightarrow(A, Z+1)+$ $e^{-}+\bar{v}_{e}$, of the fission fragments produces a large number of electron antineutrinos $\bar{\nu}_{e}$ which are emitted isotropically from the core. During the fuel "burning" process, uranium isotopes breed ${ }^{239} \mathrm{Pu}$ and ${ }^{241} \mathrm{Pu}$. The latter may in addition to ${ }^{235} \mathrm{U}$ undergo a fission process only with slow (thermal) neutrons, while ${ }^{238} \mathrm{U}$ is fissionable by fast neutrons only. The decay of ${ }^{239} \mathrm{Pu}$ produces substantially less antineutrinos than the decay of ${ }^{235} \mathrm{U}$ in the same energy range; therefore, during a typical reactor fuel cycle, the amount of antineutrinos decreases as uranium content decreases and the concentration of plutonium increases.

Each fission releases on average $\sim 200 \mathrm{MeV}$ of energy and produces $\sim 6 \bar{v}_{e}(\sim 3$ beta decays per fission fragment), with energies below $\sim 10 \mathrm{MeV}$. This sets the number of antineutrinos emitted by a typical reactor to $\sim 2 \times 10^{20} \bar{\nu}_{e} / s$ per GWth of thermal power. The energy spectrum of the antineutrinos depends on the fuel composition at a given time $\left({ }^{239} \mathrm{Pu}\right.$ antineutrinos are slightly less energetic than those of ${ }^{235} \mathrm{U}$ fission products).

The production of reactor antineutrinos is not exclusively through beta decay of the fission fragments of the four main fissile isotopes $\left({ }^{235} \mathrm{U},{ }^{238} \mathrm{U},{ }^{239} \mathrm{Pu}\right.$, and $\left.{ }^{241} \mathrm{Pu}\right)$. The neutron capture in ${ }^{238} \mathrm{U}(n, \gamma){ }^{239} \mathrm{U}$ also generates these particles in a process that contributes about $17 \%$ of the total antineutrino flux. This process occurs when a nucleus of ${ }^{238} \mathrm{U}$ captures a neutron, leading to the following reaction: ${ }^{238} \mathrm{U}+n \rightarrow$ ${ }^{239} \mathrm{U} \rightarrow{ }^{239} \mathrm{~Np} \rightarrow{ }^{239} \mathrm{Pu}$, and it produces two antineutrinos through two beta decays of ${ }^{239} \mathrm{U}$. This process occurs at a rate of $\sim 0.6$ per fission and produces antineutrinos with energies below $1.3 \mathrm{MeV}$.

Let us now consider the antineutrinos emitted by one of the reactors at the LVNPP. Ignoring the neutron capture contribution, the antineutrino flux $\Phi_{i}$ above $1.8 \mathrm{MeV}$ can be calculated from the fission rates $f_{i}(t)$ and the antineutrino energy spectrum $S_{i}\left(E_{\bar{v}}\right)$ of each isotope $i$, where $i=1,2,3,4$ corresponds to each of the main fissile isotopes of the reactor core: ${ }^{235} \mathrm{U},{ }^{238} \mathrm{U},{ }^{239} \mathrm{Pu}$, and ${ }^{241} \mathrm{Pu}$.

Assuming neutrinos are emitted isotropically, using a sphere of radius $R$, the flux at a time $t$ for each isotope $i$ is calculated as

$$
\Phi_{i}\left(E_{\bar{v}}, t\right)=\frac{1}{4 \pi R^{2}} S_{i}\left(E_{\bar{\nu}}\right) f_{i}(t),
$$

with $R$ being the distance from the reactor core to the detector.

In our calculation of the antineutrino flux, we have used the new predictions of the energy spectra including the effect of the reactor antineutrino anomaly, as reported by $[27,35$, 36]. The antineutrino energy spectra $S_{i}\left(E_{\bar{v}}\right)$ between 1.8 and

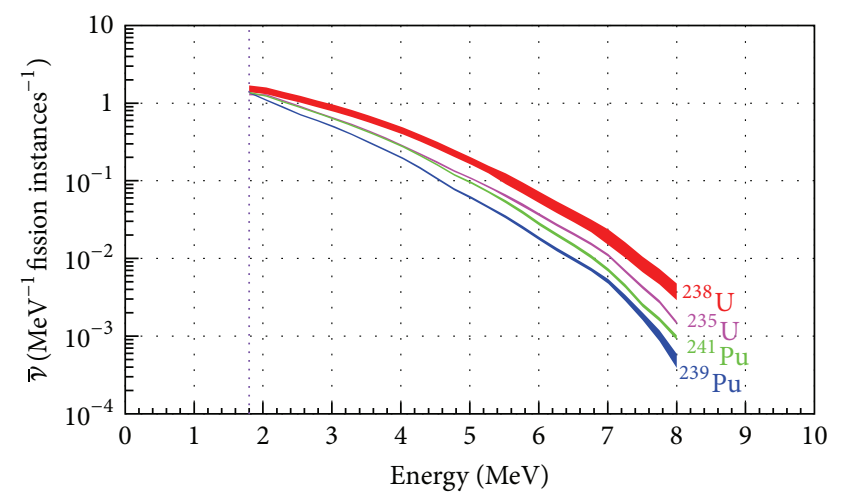

FIGURE 5: Energy spectra of the emitted antineutrinos per fission of ${ }^{235} \mathrm{U},{ }^{238} \mathrm{U},{ }^{239} \mathrm{Pu}$, and ${ }^{241} \mathrm{Pu}$. The dotted line at $1.8 \mathrm{MeV}$ indicates the threshold for the Inverse Beta Decay (IBD) process.

$8 \mathrm{MeV}$ are shown in Figure 5. For the purposes of reactor monitoring, the effect of this anomaly can be ignored as long as only comparisons between the relative changes of the spectrum throughout the reactor operation cycle are made.

Figure 6 shows the antineutrino flux for each of the main fissile isotopes of one of the reactors in the LVNPP, at day 5 (a) and then at day 200 (b); in the latter, the flux can be considered stable. The total flux (the sum of the four isotopes) for each time period is also shown in the gray curve in this figure. The total flux at day 200 is shown in Table 4.

Two systematic uncertainties are associated with the calculation of the antineutrino flux: the error due to the energy spectra of antineutrinos $\delta\left(S\left(E_{\bar{v}}\right)\right)$ and an error $\delta\left(P_{\mathrm{th}}\right)$ associated with fluctuations in the thermal power of the reactor core, assumed to be $5 \%$ along one fuel cycle. The total error is the sum in quadrature of these uncertainties. The uncertainties in the antineutrino spectra are taken from $[35,36]$ and are of the order of $2-5 \%$ for the dominant parts of the spectra from ${ }^{235} \mathrm{U},{ }^{239} \mathrm{Pu}$, and ${ }^{239} \mathrm{Pu}$ and between 10 and $17 \%$ for the dominant part of the spectrum from ${ }^{238} \mathrm{U}$.

The total flux per reactor at day 200 from our calculation is compared in Figure 7 to that obtained according to the parametrization suggested by Vogel and Engel [18], setting the fission rates of the four main fissile isotopes to those output by the simulation $\left(2.7 \times 10^{19}\right.$ fis/s for ${ }^{235} \mathrm{U}, 7.7 \times$ $10^{18} \mathrm{fis} / \mathrm{s}$ for ${ }^{238} \mathrm{U}, 2.8 \times 10^{19} \mathrm{fis} / \mathrm{s}$ for ${ }^{239} \mathrm{Pu}$, and $1.5 \times 10^{18} \mathrm{fis} / \mathrm{s}$ for ${ }^{241} \mathrm{Pu}$ ). The two calculations agree well within the assumed uncertainties.

Spent fuel is stored temporarily in a nearby place to the reactor core and is expected to have a small contribution $(<1 \%)$ to the antineutrino flux above the IBD reaction threshold [37] and was not considered for the calculation in this work.

\section{Event Rates}

Although the IBD reaction has a very small cross section $\left(\sim 10^{-43} \mathrm{~cm}^{2}\right)$, the enormous flux emitted by a nuclear reactor allows the signal to be observed with a relatively small detector located at a relatively short distance from the reactor 


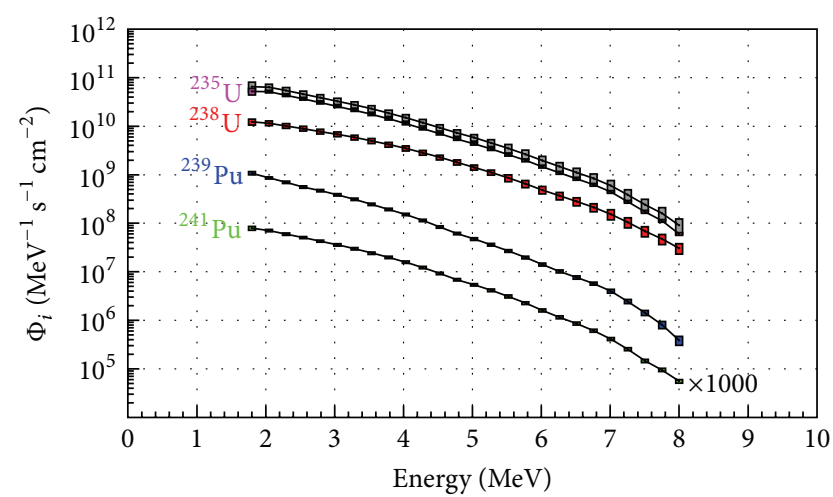

(a)

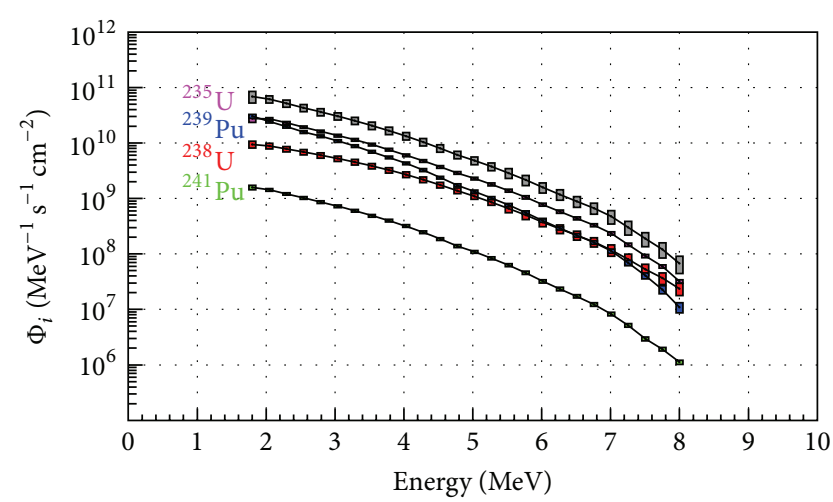

(b)

Figure 6: Antineutrino flux produced by the fission of the 4 main fissile isotopes at 100 meters from one of the LVNPP reactors of 2.027 GWth at days 5 (a) and 200 (b) of operation. The gray curve (a) is the total flux.

TABLE 4: Total antineutrino flux at day 200 for one of the reactors of the LVNPP, at $100 \mathrm{~m}$ from the reactor core. The last column shows the total systematic error $\delta$ (sys).

\begin{tabular}{|c|c|c|}
\hline$E[\mathrm{MeV}]$ & $\Phi_{\text {total }}\left[\mathrm{MeV}^{-1} \mathrm{~s}^{-1} \mathrm{~cm}^{-2}\right]$ & $\delta$ (sys) [\%] \\
\hline 1.800 & $6.921 E+10$ & 7.2 \\
\hline 2.048 & $6.192 E+10$ & 3.6 \\
\hline 2.296 & $5.185 E+10$ & 3.6 \\
\hline 2.544 & $4.304 E+10$ & 3.7 \\
\hline 2.792 & $3.634 E+10$ & 3.7 \\
\hline 3.040 & $3.052 E+10$ & 3.7 \\
\hline 3.288 & $2.542 E+10$ & 3.7 \\
\hline 3.536 & $2.066 E+10$ & 3.8 \\
\hline 3.784 & $1.671 E+10$ & 3.8 \\
\hline 4.032 & $1.336 E+10$ & 3.9 \\
\hline 4.280 & $1.045 E+10$ & 4.0 \\
\hline 4.528 & $8.026 E+09$ & 4.0 \\
\hline 4.776 & $6.147 E+09$ & 4.1 \\
\hline 5.024 & $4.841 E+09$ & 4.2 \\
\hline 5.272 & $3.761 E+09$ & 4.3 \\
\hline 5.520 & $2.871 E+09$ & 5.2 \\
\hline 5.768 & $2.157 E+09$ & 5.4 \\
\hline 6.016 & $1.584 E+09$ & 5.5 \\
\hline 6.264 & $1.179 E+09$ & 5.6 \\
\hline 6.512 & $8.900 E+08$ & 5.8 \\
\hline 6.760 & $6.692 E+08$ & 5.9 \\
\hline 7.008 & $4.752 E+08$ & 7.0 \\
\hline 7.256 & $3.027 E+08$ & 7.4 \\
\hline 7.504 & $1.881 E+08$ & 7.8 \\
\hline 7.752 & $1.210 E+08$ & 8.4 \\
\hline 8.000 & $6.763 E+07$ & 9.8 \\
\hline
\end{tabular}

core (several tens of meters). The total IBD cross section as a function of the neutrino energy is shown in Figure 7 (right scale). Here we consider 1 ton of polyvinyl-toluene (PVT) plastic scintillator located at $100 \mathrm{~m}$ from each of the reactor cores, similar to the detector considered in $[5,6]$.

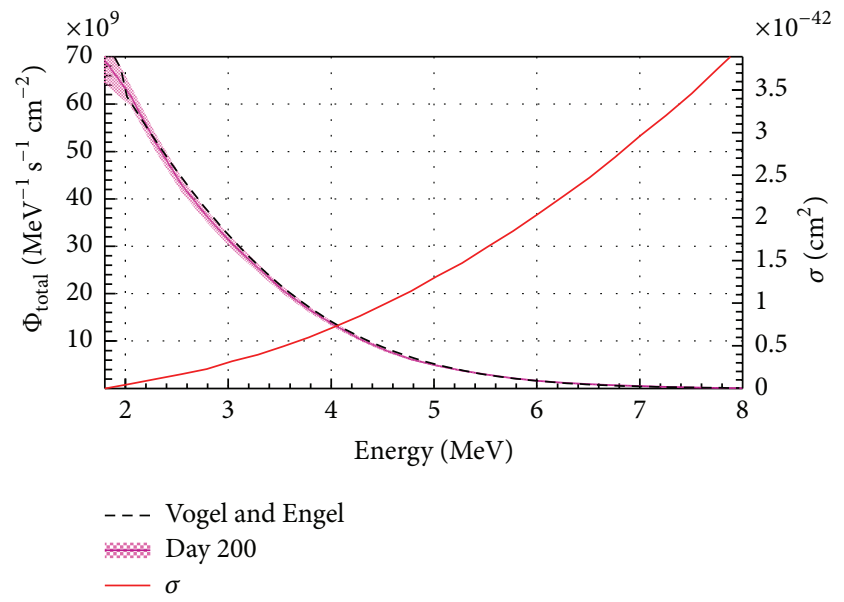

FIgURE 7: Total antineutrino flux per reactor at day 200 (solid curve with error band). The error includes the sum of the relative uncertainties of each of the 4 main fissile isotopes in the reactor. The dashed curve shows the parametrization suggested in [18] with the fission rates calculated by DRAGON for the LVNPP. The continuous line shows the IBD cross section as given by [19].

This segmented detector is a one-cubic-meter array of 100 optically isolated plastic scintillator bars of dimensions $100 \mathrm{~cm} \times 10 \mathrm{~cm} \times 10 \mathrm{~cm}(10 \mathrm{~kg}$ per bar), instrumented with photomultiplier tubes (PMTs) at both ends and separated with sheets coated with gadolinium oxide $\left(\mathrm{Gd}_{2} \mathrm{O}_{3}\right)$. The $100 \mathrm{~m}$ distance was chosen from aerial photographs of the LVNPP site, so as to place the detector equidistantly from both reactor cores, in an existing open space away from any discernible infrastructure.

Following the IBD interaction (see (1)) of an antineutrino with a proton in the plastic scintillator, the prompt positron and the delayed neutron capture are detected independently. The positron will deposit its energy (comparable to the incident neutrino energy) through ionization in the scintillator and annihilate producing two gamma rays. The neutron will thermalize in the plastic and will eventually be captured by a gadolinium nucleus in one of the $\mathrm{Gd}_{2} \mathrm{O}_{3}$-coated sheets, with 


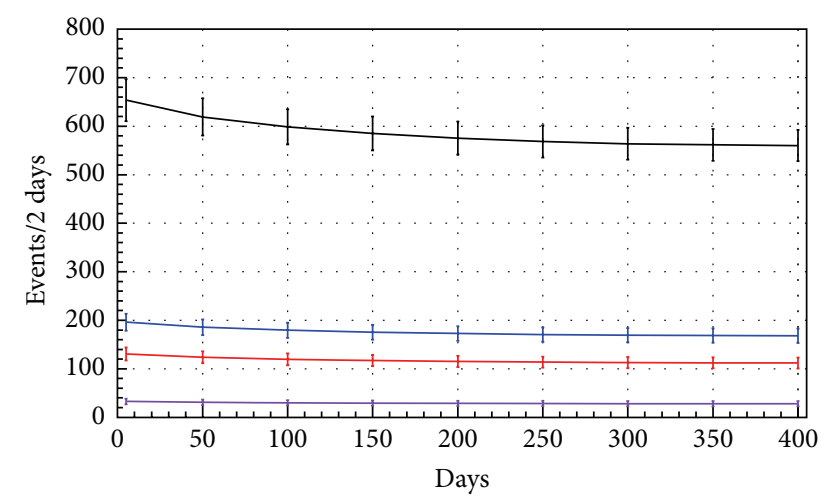

FigURE 8: Number of events accumulated over 2 days of exposure along a complete cycle of 400 days at LVNPP with two reactors in 1 ton of plastic scintillator. Curves for detection efficiencies (lower to higher) of $5 \%, 20 \%, 30 \%$, and $100 \%$ are shown.

a typical capture time of several tens of $\mu$ s, liberating a cascade of gamma rays with a total energy of $\sim 8 \mathrm{MeV}$. Recording of the energy deposition in each bar allows for the implementation of powerful background rejection techniques $[5,6]$ which are unattainable with single-volume detectors.

The number of IBD interactions associated with neutrinos from isotope $i$, emitted with energies between $E_{\text {min }}$ and $E_{\max }$, and occurring in a time interval $\Delta t\left(\Delta N_{\mathrm{ev}(i)}\right)$ is calculated by integrating the product of the flux $\Phi_{i}$, the IBD cross section $\sigma\left(E_{\bar{\gamma}}\right)$ [19], and the number of targets (in this case free protons) $N_{p}$, over the energy interval:

$$
\frac{\Delta N_{\mathrm{ev}(i)}}{\Delta t}=N_{p} \int_{E_{\min }}^{E_{\max }} \Phi_{i}\left(E_{\bar{\gamma}}, t\right) \sigma\left(E_{\bar{\gamma}}\right) d E_{\bar{\gamma}} .
$$

Depending on the characteristics of the detector, only a fraction of these interactions will be observed. For a simple estimate, let this fraction be a uniform efficiency factor $\epsilon$. For neutrinos coming from nuclear reactors, $E_{\min }$ is the threshold of $1.8 \mathrm{MeV}$ and $E_{\max }$ is $\sim 10 \mathrm{MeV}$. The number of detected events for each isotope is then approximated by

$$
N_{\mathrm{ev}(i)}=\epsilon \times N_{p} \frac{\Delta t}{4 \pi R^{2}} \int_{E_{\min }}^{E_{\max }} S_{i}\left(E_{\bar{\nu}}\right) f_{i}(t) \sigma\left(E_{\bar{v}}\right) d E_{\bar{\nu}}
$$

where $\Delta t$ is a small exposure time compared with the time scale in which fission rates $f_{i}(t)$ change appreciably. Calculation for longer times requires integration over time.

Finally, the total number of events is equal to the sum of the events for each isotope:

$$
N_{\mathrm{ev}(\text { total })}=\sum_{i=1}^{4} N_{\mathrm{ev}(i)}
$$

These are shown in Figure 8, considering four different detector efficiencies $(5 \%, 20 \%, 30 \%$, and $100 \%)$ and counting for two consecutive days.

The authors of $[5,6]$ estimate an antineutrino detection efficiency of $9.24 \%$ and an antineutrino event rate of 147 events/day in a 1-ton detector with an uncertainty of the order of $30 \%$. This is consistent, within errors, with our expected rate in a similar detector, from Figure 8, of 280 events/day with the two reactors and $100 \%$ efficiency (stable after day 350), after scaling for the difference in reactor power and distance and assuming the same efficiency.

For our background estimate, we assume a similar analysis technique to $[5,6]$. The authors measured an uncorrelated background, free of fast neutron events, of $~ 365$ events/day with an uncertainty of $19 \%$, operating a $360 \mathrm{~kg}$ detector with the reactor OFF for 21 days.

Taking the above considerations into account, a 1-ton segmented detector of this type, with a 9.24\% signal efficiency, deployed at $100 \mathrm{~m}$ from the cores of the LVNPP reactors would expect to observe an antineutrino event rate of 26 events/day, with a background (defined as in $[5,6]$ ) of $~ 1013$ events/day. At this reactor distance, the daily rate could be measured with a significance of $5 \sigma$, provided the background rate normalization is determined with a precision of $0.5 \%( \pm 5$ events/day), which would require significant improvements in the analysis and about a couple of months of reactor OFF data. Improvements in the detector design such as adding water or polyethylene shields to moderate the fast neutrons are expected to yield more efficient background rejection.

Changes in the total antineutrino event rate over the fuel cycle are expected at the $10-15 \%$ level, corresponding to a variation of several tens of $\mathrm{kg}$ of $\mathrm{Pu}$ being produced in the reactor. Given the low statistics of the expected antineutrino signal at the proposed detector standoff with the projected efficiency, it will be desirable to add whatever spectral shape information is possible to the analysis. The capabilities of the detector in this regard will be the subject of a future work.

\section{Conclusions}

We calculated the antineutrino flux produced by the reactors at the LVNPP in México using the DRAGON simulation code in the energy range $1.8 \mathrm{MeV}<E_{v}<8 \mathrm{MeV}$. The simulation provided the time evolution of the fission rates and hence the change of the flux along the 400-day-long fuel cycle, starting with an initial load with no plutonium. A total systematic uncertainty on the flux ranging from $3.6 \%$ to $9.8 \%$, depending on the antineutrino energy, was calculated assuming a $5 \%$ uncertainty on the reactor power and the uncertainties reported for the antineutrino spectra of the dominant fissile isotopes $[35,36]$. Our calculation agrees well with frequently used parametrization [18] when used with the fission rates from the simulation of the LVNPP BWR reactors.

The simulation code required the knowledge of generic parameters of the BWR reactors, at LVNPP, available in the literature, and showed the fission rates to have moderate dependence on variations of the coolant, moderator, and fuel temperatures for a fixed thermal power.

A segmented plastic scintillator detector of the type considered in $[5,6]$ located at $100 \mathrm{~m}$ from the two reactors could in principle detect the emitted antineutrinos; however, new methods for background suppression and rejection for aboveground operation would need to be implemented. Operation at a significantly shorter distance from the cores 
must be pursued for its successful application to reactor monitoring.

\section{Conflict of Interests}

The authors declare that there is no conflict of interests regarding the publication of this paper.

\section{Acknowledgments}

The authors acknowledge the support of UNAM-PAPIIT, Grants nos. IB100413 and IN112213, and CONACYT through Grants CB-2009/00131598 and 398495 (scholarship holder number: 570620). They also especially thank Dr. Guy Marleau from École Polytechnique de Montréal and Dr. Christopher Jones from MIT for their valuable help and advice throughout the installation and implementation of the DRAGON code simulation.

\section{References}

[1] F. P. An, J. Z. Bai, A. B. Balantekin et al., "Observation of electron-antineutrino disappearance at Daya Bay," Physical Review Letters, vol. 108, no. 17, Article ID 171803, 2012.

[2] J. K. Ahn, S. Chebotaryov, J. H. Choi et al., "Observation of reactor electron antineutrinos disappearance in the RENO experiment," Physical Review Letters, vol. 108, no. 19, Article ID 191802, 6 pages, 2012.

[3] Y. Abe, C. Aberle, J. C. dos Anjos et al., "First measurement of $\theta_{13}$ from delayed neutron capture on hydrogen in the Double Chooz experiment," Physics Letters B, vol. 723, no. 1-3, pp. 6670, 2012.

[4] N. S. Bowden, A. Bernstein, S. Dazeley, R. Svoboda, A. Misner, and T. Palmer, "Observation of the isotopic evolution of pressurized water reactor fuel using an antineutrino detector," Journal of Applied Physics, vol. 105, no. 6, Article ID 064902, 2009.

[5] Y. Kuroda, S. Oguri, Y. Kato et al., "A mobile antineutrino detector with plastic scintillators," Nuclear Instruments and Methods in Physics Research Section A: Accelerators, Spectrometers, Detectors and Associated Equipment, vol. 690, pp. 41-47, 2012.

[6] S. Oguri, Y. Kuroda, Y. Kato et al., "Reactor antineutrino monitoring with a plastic scintillator array as a new safeguards method," Nuclear Instruments and Methods in Physics Research, Section A: Accelerators, Spectrometers, Detectors and Associated Equipment, vol. 757, pp. 33-39, 2014.

[7] A. Porta, "Reactor neutrino detection for non proliferation with the Nucifer experiment," Journal of Physics: Conference Series, vol. 203, Article ID 012092, 2010.

[8] E. Christensen, P. Huber, P. Jaffke, and T. E. Shea, "Antineutrino monitoring for heavy water reactors," Physical Review Letters, vol. 113, no. 4, Article ID 042503, 2014.

[9] E. Casimiro and J. C. Anjos, "Cosmic muon background and reactor neutrino detectors: the Angra experiment," Journal of Physics: Conference Series, vol. 116, no. 1, Article ID 012003, 2008.

[10] V. Belov, V. Brudanin, M. Danilov et al., "Registration of reactor neutrinos with the highly segmented plastic scintillator detector DANSSino," Journal of Instrumentation, vol. 8, no. 5, Article ID P05018, 2013.
[11] IAEA, Final Report: Focused Workshop on Antineutrino Detection for Safeguards Applications, International Atomic Energy Agency, Vienna, Austria, 2008.

[12] N. Harms and P. Rodriguez, "Safeguards at light-water reactors-current practices, future directions," IAEA Bulletin, vol. 38, no. 4, p. 16, 1996, http://www.iaea.org/Publications/ Booklets/Teaming-Inspectors/index.html.

[13] A. Bernstein, Y. Wang, G. Gratta, and T. West, "Nuclear reactor safeguards and monitoring with antineutrino detectors," Journal of Applied Physics, vol. 91, no. 7, article 4672, 2002.

[14] M. M. Nieto, A. C. Hayes, C. M. Teeter, W. B. Wilson, and W. D. Stanbro, "Detection of antineutrinos for non-proliferation," Nuclear Science and Engineering, vol. 149, 3, pp. 270-276, 2005.

[15] E. Christensen, P. Huber, and P. Jaffke, "Antineutrinoreactor safeguards—a case study," http://arxiv.org/abs/1312.1959v2.

[16] V. Bulaevskaya and A. Bernstein, "Detection of anomalous reactor activity using antineutrino count evolution over the course of a reactor cycle," Journal of Applied Physics, vol. 109, no. 11, Article ID 114909, 2011.

[17] Qué es el ciclo del combustible nuclear?, Booklet edited by: CFE, F-65.

[18] P. Vogel and J. Engel, "Neutrino electromagnetic form factors," Physical Review D, vol. 39, no. 11, article 3378, 1989.

[19] P. Vogel and J. F. Beacom, "Angular distribution of neutron inverse beta decay, $\bar{v}_{e}+\vec{p} e^{+}+n$," Physical Review $D$, vol. 60 , no. 5, Article ID 053003, 1999.

[20] C. L. Jones, A. Bernstein, J. M. Conrad et al., "Reactor simulation for antineutrino experiments using DRAGON and MURE," Physical Review D, vol. 86, no. 1, Article ID 012001, 2012.

[21] C. O. Muehlhause and S. Oleksa, "Antineutrino flux from a reactor," Physical Review, vol. 105, no. 4, pp. 1332-1334, 1957.

[22] A. I. Afonin, S. A. Bogatov, A. A. Borovoi et al., "Search for neutrino oscillations in an experiment in the reactor of the Rovno nuclear power plant," Pis'ma $v$ Zhurnal Èksperimental'noi i Teoreticheskoi Fiziki, vol. 42, no. 5, pp. 230-233, 1985.

[23] G. Zacek, F. V. Feilitzsch, R. L. Mössbauer et al., "Neutrinooscillation experiments at the Gösgen nuclear power reactor," Physical Review D, vol. 34, no. 9, pp. 2621-2636, 1986.

[24] Y. Declais, H. de Kerret, B. Lefièvre et al., "Study of reactor antineutrino interaction with proton at Bugey nuclear power plant," Physics Letters B, vol. 338, no. 2-3, pp. 383-389, 1994.

[25] V. I. Kopeikin, L. A. Mikaelyan, and V. V. Sinev, "Spectrum of electronic reactor antineutrinos," Physics of Atomic Nuclei, vol. 60, no. 2, pp. 172-176, 1997.

[26] M. Apollonio, A. Baldini, C. Bemporad et al., "Search for neutrino oscillations on a long base-line at the $\mathrm{CHOOZ}$ nuclear power station," The European Physical Journal C-Particles and Fields, vol. 27, no. 3, pp. 331-374, 2003.

[27] G. Mention, M. Fechner, T. Lasserre et al., "Reactor antineutrino anomaly," Physical Review D, vol. 83, no. 7, Article ID 073006, 2011.

[28] J. Cao, "Determining reactor neutrino flux," Nuclear Physics BProceedings Supplements, vol. 229-232, pp. 205-209, 2012.

[29] K. Nakajima, K. Inoue, K. Owada et al., "A simple model of reactor cores for reactor neutrino flux calculations for the KamLAND experiment," Nuclear Instruments and Methods in Physics Research, Section A: Accelerators, Spectrometers, Detectors and Associated Equipment, vol. 569, no. 3, pp. 837-844, 2006.

[30] IAEA, Energy, Electricity and Nuclear Power Estimates for the Period up to 2050, Reference Data Series no. 1, IAEA, Vienna, Austria, 2014. 
[31] G. Marleau, R. Roy, and A. Hébert, "DRAGON: a collision probability transport code for cell and supercell calculations," Report IGE-157, Institut de Génie Nucléaire, École Polytechnique de Montréal, Montréal, Canada, 1994, http://www.polymtl.ca/ nucleaire/DRAGON/en/.

[32] Modifications to the Installation of DRAGON, http://dspace .mit.edu/handle/1721.1/70045.

[33] X. B. Ma, F. Lu, L. Z. Wang, Y. X. Chen, W. L. Zhong, and F. P. An, "Uncertainties analysis of fission fraction for reactor antineutrino experiments using DRAGON," http://arxiv.org/abs/1405 .6807.

[34] H. H. López, "Fuel assembly with inert matrix fuel rods as reload options for Laguna Verde NPP," Annals of Nuclear Energy, vol. 40, no. 1, pp. 215-220, 2012.

[35] M. Fallot, S. Cormon, M. Estienne et al., "New antineutrino energy spectra predictions from the summation of beta decay branches of the fission products," Physical Review Letters, vol. 109, no. 20, Article ID 202504, 2012.

[36] T. A. Mueller, D. Lhuillier, M. Fallot et al., "Improved predictions of reactor antineutrino spectra," Physical Review C, vol. 83, no. 5, Article ID 054615, 17 pages, 2011.

[37] V. I. Kopeikin, L. A. Mikaelyan, and V. V. Sinev, "Antineutrino background from spent-fuel storage in sensitive searches for $\theta_{13}$ at reactors," Physics of Atomic Nuclei, vol. 69, no. 2, pp. 185-188, 2006. 

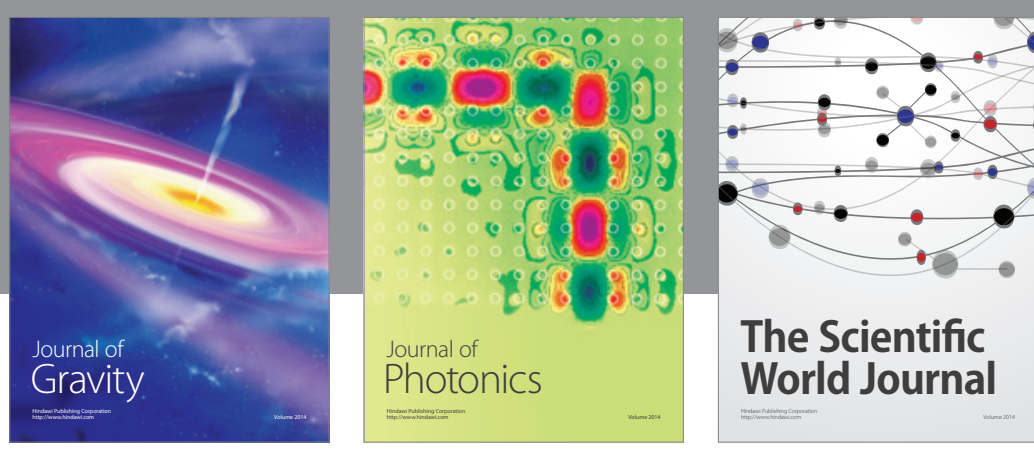

The Scientific World Journal
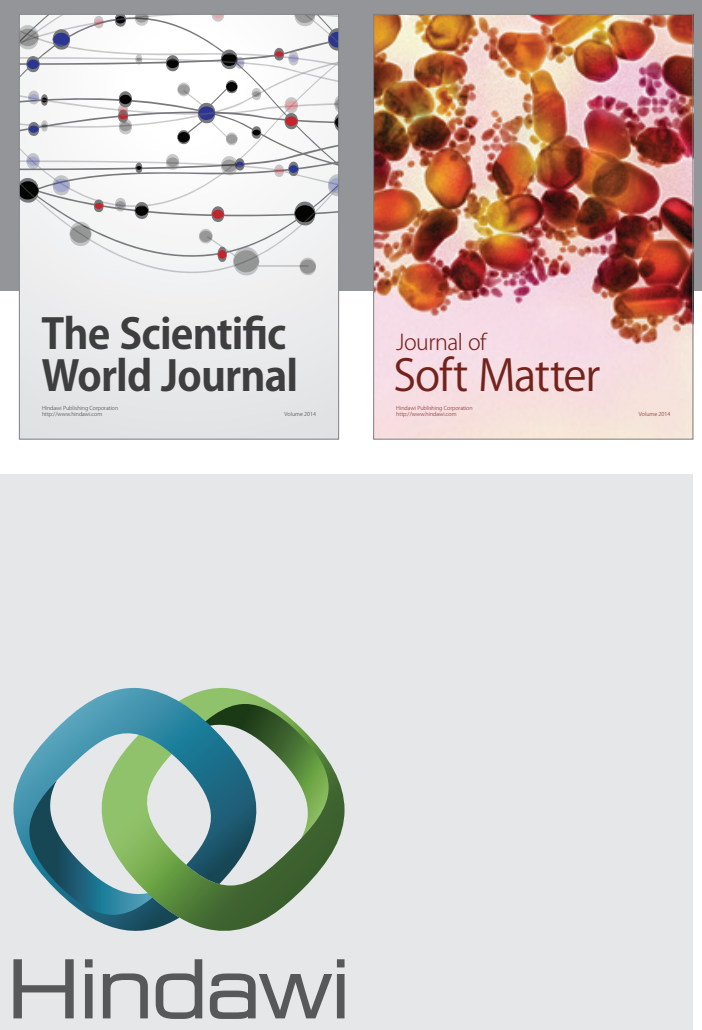

Submit your manuscripts at

http://www.hindawi.com

nternational Journal of

Statistical Mechanics
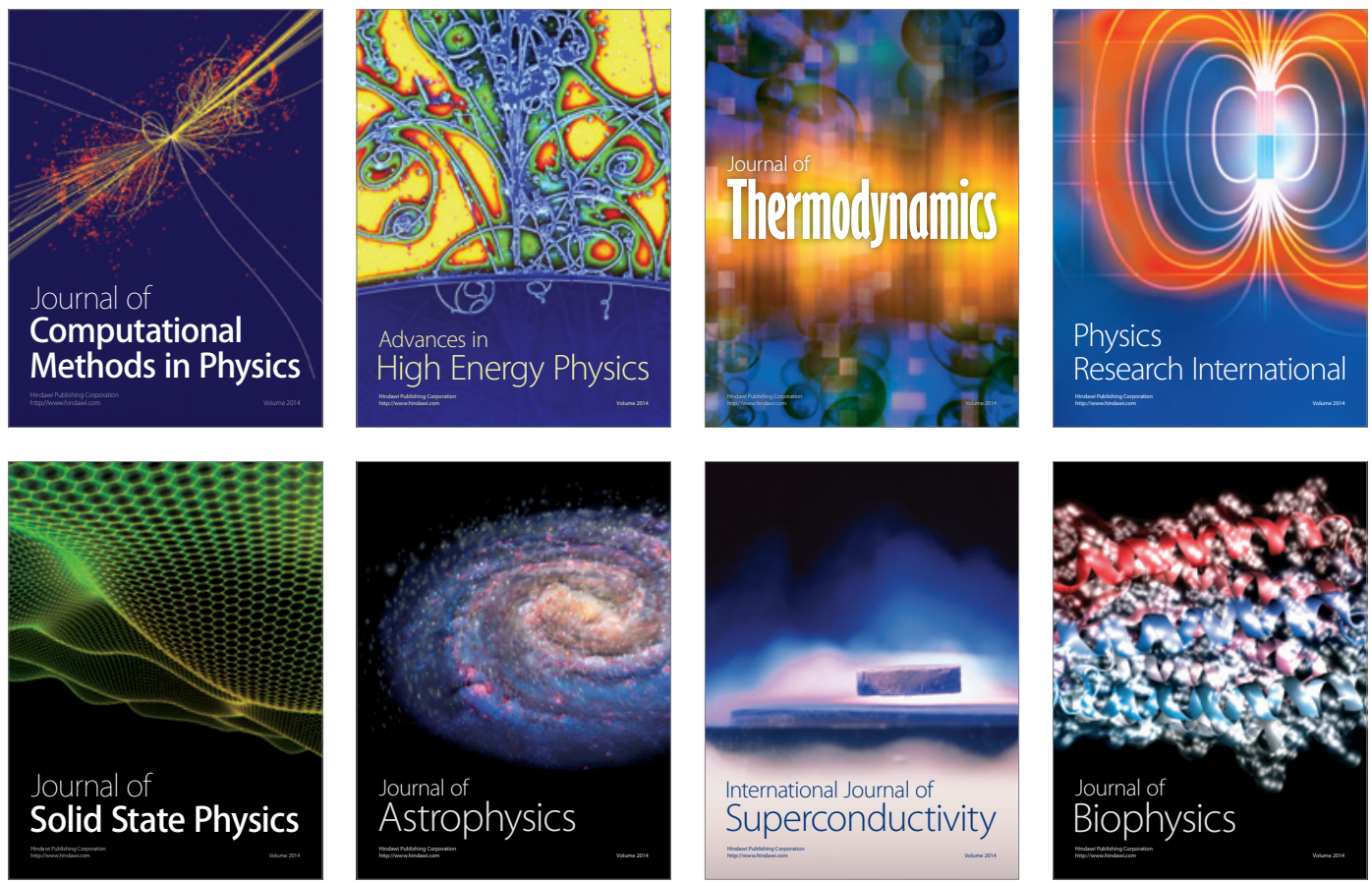
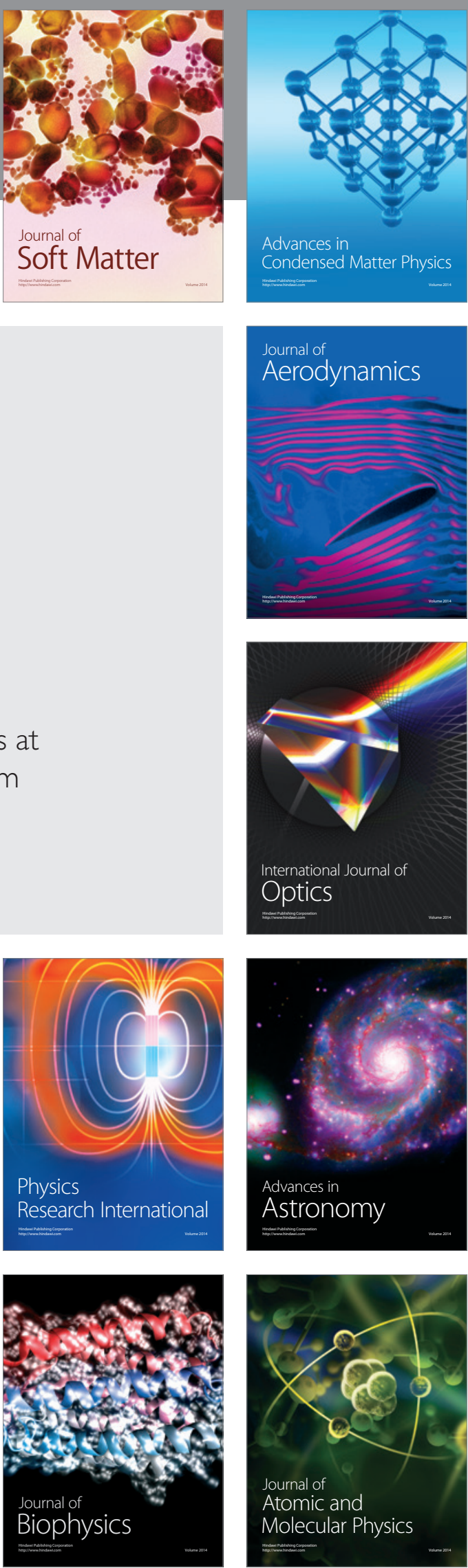